\title{
Characteristic Impedance Analysis of Medium-Voltage Underground Cables with Grounded Shields and Armors for Power Line Communication
}

\author{
Hongshan Zhao *, Weitao Zhang $(\mathbb{D}$ and Yan Wang \\ School of Electrical and Electronic Engineering, North China Electric Power University, Baoding 071003, China; \\ almightywiz@outlook.com (W.Z.); wang_yan0421@163.com (Y.W.) \\ * Correspondence: zhaohshcn@ncepu.edu.cn; Tel.: +86-135-0313-5810
}

Received: 25 March 2019; Accepted: 18 May 2019; Published: 23 May 2019

check for updates

\begin{abstract}
The characteristic impedance of a power line is an important parameter in power line communication (PLC) technologies. This parameter is helpful for understanding power line impedance characteristics and achieving impedance matching. In this study, we focused on the characteristic impedance matrices (CIMs) of the medium-voltage (MV) cables. The calculation and characteristics of the CIMs were investigated with special consideration of the grounded shields and armors, which are often neglected in current research. The calculation results were validated through the experimental measurements. The results show that the MV underground cables with multiple grounding points have forward and backward CIMs, which are generally not equal unless the whole cable structure is longitudinally symmetrical. Then, the resonance phenomenon in the CIMs was analyzed. We found that the grounding of the shields and armors not only affected their own characteristic impedances but also those of the cores, and the resonance present in the CIMs should be of concern in the impedance matching of the PLC systems. Finally, the effects of the grounding resistances, cable lengths, grounding point numbers, and cable branch numbers on the CIMs of the MV underground cables were discussed through control experiments.
\end{abstract}

Keywords: power line communication; medium-voltage underground cable; impedance matching; characteristic impedance matrix; partial conductor grounding

\section{Introduction}

Smart grid (SG) technologies are attracting growing attention given their capacity to sustainably manage power using intelligent grids. SG infrastructures require a bi-directional communication to enhance the energy efficiency, reliability and safety of the electricity systems [1]. When exploring SG communication systems, a universal data and network communications protocol is essential to achieve an interoperable environment [2]. In this respect, IEC 61850, an object-oriented standard, is considered the foundation of the SG communications systems [3,4]. The communication medium used in the communication system is another pivotal issue. Given the wide range of costs, latency, and bandwidth requirements of smart grid communications, a communication system that integrates multiple communication mediums is desirable $[5,6]$. Therefore, many communication technologies have since been evaluated and compared [6,7]. Among them, power line communication (PLC) is considered a feasible alternative that has attracted significant interest, and it has been gradually applied in advanced measurement systems and in remote fault detection systems [8,9]. This is due to its low cost, convenient deployment, and wide coverage compared with the high data capability communication technologies (e.g., fiber-optic ethernet) [6,10]. 
However, power line networks have numerous branches, especially in the medium-voltage (MV) and low-voltage (LV) networks, and their network typologies and loads may change unpredictably and extremely, which makes the impedance characteristics of power lines complicated and changeable, thereby increasing the difficulty of impedance matching in PLC systems. The methods of impedance matching mainly include characteristic impedance matching and complex conjugate matching. The purpose of characteristic impedance matching is to reduce or eliminate the reflected components of the carrier signal to increase the signal voltage at the receivers and to improve the signal-to-noise ratio (SNR) [11-13]. This method requires impedance matching between the internal impedances of the receivers and the characteristic impedances of the power lines. The purpose of complex conjugate matching is to adapt the internal impedance of the transceivers to the changeable access impedance of the power lines to achieve maximum power transmission [14-16]. Additionally, some optimization methods based on access impedance have been proposed to increase the data rate and channel capacity of the PLC systems $[17,18]$. Here, the access impedances of the power lines are closely related to their characteristic impedances $[19,20]$. Therefore, the characteristic impedances of the power lines are important in the analysis of the impedance characteristics and in the realization of impedance matching.

The power lines of interest in this paper are MV underground cables, which are employed in almost all new urban and suburban installations. The current studies on their characteristic impedances and impedance matching are mainly based on the two-conductor transmission line model $[19,21]$. However, the MV underground cables have an important multiconductor nature, meaning that the signal coupling between the conductors cannot be ignored, and thus the impedance matching at the terminals of the power lines requires impedance matching at any port comprised of two power lines [13]. At this point, it is necessary to analyze the characteristic impedance matrices (CIMs) of the MV underground cables.

In the MV underground cable networks, the shields and armors are grounded at the ring main units (RMUs), where the cable branches exist. The grounding points may considerably affect the PLC channel characteristics [22-24]. Similar issues have been studied for overhead lines when the shield wires are grounded at towers, and different treatments have been adopted. A typical approach involves assuming that the shield wires are continuously grounded and that the shield wires have zero potential along their entire length [25-29]. In previous studies [30-32], this assumption was proven to be unsuitable when the electrical length between towers is odd multiples of a one-half wavelength of signal. In addition, these studies mainly investigated the signal attenuation based on transmission and admittance matrices. For the characteristic impedances of power lines, a simplified method involves ignoring the mutual inductance between the ungrounded and grounded conductors, so that their characteristic impedances are considered separately, despite this method being applicable in certain special cases [33,34]. In Reference [35], for the first time, the CIMs of overhead lines with shield wires that were periodically grounded were calculated under a lossless assumption. In other studies [36,37], the CIMs of typical high-voltage (HV) lossy overhead lines with periodically grounded and sectionalized shield wires were analyzed under an ideal grounding assumption (i.e., grounding points had zero potential), and the properties of the CIMs have been further investigated [37,38]. However, in current studies on the CIM of the MV underground cable, the grounded partial conductors were not considered. The related investigations are confined to the CIM of the uniform MV underground cable $[39,40]$, and the results only represent the CIM of one cable section rather than that of the whole cable structure between the PLC transceivers, which is often nonuniform owing to the grounded shields and armors. The latter is more important in reducing or eliminating the reflected signal at the receivers or transmitters [36].

In this study, we focused on MV underground cables with grounded shields and armors, where the CIMs were calculated and analyzed after considering the multiconductor and non-uniformity characteristics of the cables. Simultaneously, the high-frequency loss and non-ideal grounding conditions (i.e., the shields and armors were grounded only at the RMUs, and the grounding points potential was non-zero) were considered. 
The remainder of this paper is organized as follows. Section 2 details the parameter matrices and multiconductor transmission line representations of the MV underground cables. Section 3 is dedicated to calculating the CIMs of the MV underground cables with grounded shields and armors, and the results are validated through experimental measurements in Section 4. Then, the characteristics of the CIMs and the effects of the network structures and parameters are discussed in Section 5. The conclusions are provided in Section 6.

\section{Multiconductor Transmission Lines Equations}

In this paper, the investigation of the CIM of the MV underground cable is based on multi-conductor transmission lines theory. Therefore, this section presents the geometry of MV underground cable and the basic multi-conductor transmission line representations.

At present, the MV $(10 \mathrm{kV}-35 \mathrm{kV})$ underground cable that is used widely in China is a three-phase cable, which has a cyclic-symmetric structure. The $10 \mathrm{kV}$ underground cables that were examined in this paper were the YJV22-type cables with a sectional area of $3 \times 240 \mathrm{~mm}^{2}$. The layout of this type of cable is depicted in Figure 1a. The original geometric and electromagnetic parameters of the cable are provided in Appendix A. The cable was located $3 \mathrm{~m}$ below the earth surface, where the soil resistivity is $100 \Omega \cdot m$.

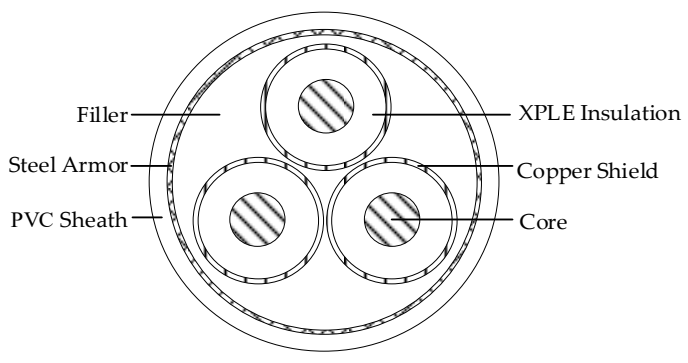

(a)

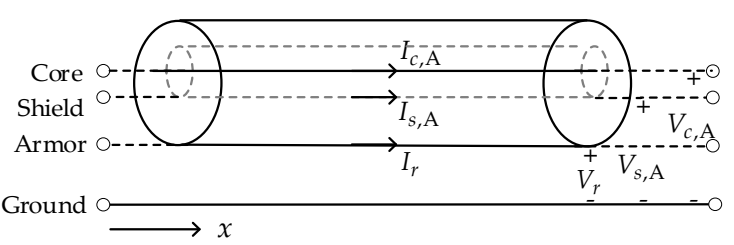

(b)

Figure 1. (a) Structure of the medium-voltage underground cable. XPLE stands for cross-linked polyethylene, PVC stands for polyvinyl chloride; (b) Voltage and current definitions of the A-phase core, A phase shield, and the armor.

The MV underground cable includes 7 conductors with three-phase cores, three-phase shields and the outermost armor. They can be treated as the uniform multiconductor transmission lines, based on the telegraph equations in the frequency domain:

$$
\left\{\begin{array}{l}
\frac{\mathrm{d}}{\mathrm{d} x} \boldsymbol{V}(x)=-\mathbf{Z I}(x) \\
\frac{\mathrm{d}}{\mathrm{d} x} \boldsymbol{I}(x)=-\boldsymbol{V} \boldsymbol{V}(x)
\end{array}\right.
$$

where $x$ denotes the position at the conductors. $Z$ and $Y$ are the per unit length (p.u.l.) impedance matrix and admittance matrix of the MV underground cable, respectively. The $7 \times 1$ vectors $V$ and $I$ represent the voltage and current in the phasor form, respectively. In this work, $V$ and $I$ are expressed as follows:

$$
\begin{gathered}
\boldsymbol{V}=\left[\begin{array}{ccccccc}
V_{c, \mathrm{~A}} & V_{s, \mathrm{~A}} & V_{c, \mathrm{~B}} & V_{s, \mathrm{~B}} & V_{c, \mathrm{C}} & V_{s, \mathrm{C}} & V_{r}
\end{array}\right]^{\mathrm{T}}, \\
\boldsymbol{I}=\left[\begin{array}{lllllll}
I_{c, \mathrm{~A}} & I_{s, \mathrm{~A}} & I_{c, \mathrm{~B}} & I_{s, \mathrm{~B}} & I_{c, \mathrm{C}} & I_{s, \mathrm{C}} & I_{r}
\end{array}\right]^{\mathrm{T}} .
\end{gathered}
$$

where $[\cdot]^{\mathrm{T}}$ denotes the matrix transpose. $V_{c, \mathrm{~A}}, V_{c, \mathrm{~B}}$ and $V_{c, \mathrm{C}}$ are the voltages of the $\mathrm{A}, \mathrm{B}$ and $\mathrm{C}$ phase cores, respectively. $V_{s, \mathrm{~A}}, V_{s, \mathrm{~B}}$ and $V_{s, \mathrm{C}}$ are the voltages of the $\mathrm{A}, \mathrm{B}$ and $\mathrm{C}$ phase shields, respectively. $V_{r}$ is the voltage of the armor. $I_{c, \mathrm{~A}}, I_{c, \mathrm{~B}}$ and $I_{c, \mathrm{C}}$ are the currents in the $\mathrm{A}, \mathrm{B}$ and $\mathrm{C}$ phase cores respectively. $I_{s, \mathrm{~A}}, I_{s, \mathrm{~B}}$ and $I_{s, \mathrm{C}}$ are the currents in the $\mathrm{A}, \mathrm{B}$ and $\mathrm{C}$ phase shields, respectively. $I_{r}$ is the current in the armor. The positive directions of voltages and currents are shown in Figure $1 \mathrm{~b}$ taking the A phase and the armor as examples. 
Apart from the filler, armor and sheath, the MV underground cable can be considered as a system that contains three subsystems, and each subsystem is comprised of the core, insulation and shield. Therefore, the p.u.l. impedance matrix $\boldsymbol{Z}$ and the admittance matrix $\boldsymbol{Y}$ can be expressed in the forms:

$$
\begin{aligned}
& {\left[\begin{array}{cccc}
Z_{A} & Z_{A B} & Z_{A C} & Z_{A r}^{T} \\
Z_{A B} & Z_{B} & Z_{B C} & Z_{B r}^{T} \\
Z_{A C} & Z_{B C} & Z_{C} & Z_{C r}^{T} \\
Z_{A r} & Z_{B r} & Z_{C r} & Z_{r}
\end{array}\right],} \\
& {\left[\begin{array}{cccc}
Y_{A} & \boldsymbol{Y}_{A B} & Y_{A C} & \boldsymbol{Y}_{A r}^{T} \\
\boldsymbol{Y}_{A B} & \boldsymbol{Y}_{B} & \boldsymbol{Y}_{B C} & \boldsymbol{Y}_{B r}^{T} \\
\boldsymbol{Y}_{A C} & \boldsymbol{Y}_{B C} & \boldsymbol{Y}_{C} & \boldsymbol{Y}_{C r}^{T} \\
\boldsymbol{Y}_{A r} & \boldsymbol{Y}_{B r} & \boldsymbol{Y}_{C r} & Y_{r}^{T}
\end{array}\right] .}
\end{aligned}
$$

where both the rows and columns of $\boldsymbol{Z}$ and $\boldsymbol{Y}$ are arranged in the order of the A-phase subsystem, the B-phase subsystem, the $\mathrm{C}$-phase subsystem, and the armor. There are identical meanings for the subscripts of submatrices in $Z$ and $Y$. Taking the submatrices in $Z$ as examples, the $2 \times 2$ submatrices $Z_{A}$, $Z_{B}$ and $Z_{C}$ are the self-impedance matrices of $A, B$ and $C$ phase subsystem, respectively. The element $Z_{r}$ is the self-impedance of the armor. The $2 \times 2$ submatrices $Z_{A B}, Z_{A C}$ and $Z_{B C}$ are the mutual impedance matrices of two different subsystems. The $2 \times 2$ submatrices $\boldsymbol{Z}_{A r}, \boldsymbol{Z}_{B r}$ and $\boldsymbol{Z}_{C r}$ are the mutual impedance matrices between the one-phase subsystem and the armor. Given the structural symmetry, the above submatrices have the relationships as follows

$$
\begin{gathered}
\left\{\begin{array}{c}
Z_{A}=Z_{B}=Z_{C}, \\
Z_{A B}=Z_{A C}=Z_{B C}, \\
Z_{A r}=Z_{B r}=Z_{C r} .
\end{array}\right. \\
\left\{\begin{array}{c}
Y_{A}=Y_{B}=Y_{C}, \\
Y_{A B}=Y_{A C}=Y_{B C}, \\
Y_{A r}=Y_{B r}=Y_{C r} .
\end{array}\right.
\end{gathered}
$$

The impedances and admittances matrices in Equations (6) and (7) were analyzed in References [41-45]. Each conductor has its own self-impedance, and there are mutual impedances between any two conductors. The mutual admittances exist only between directly adjacent conductors. For example, there is a mutual admittance between the core and the shield, but no mutual admittance between the core and the armor because of the shield. Furthermore, the aforementioned submatrices (taking partial submatrices for examples) can be expressed as:

$$
\begin{aligned}
& Z_{A}=\left[\begin{array}{cc}
Z_{c} & Z_{c s} \\
Z_{c s} & Z_{s}
\end{array}\right], Z_{A B}=\left[\begin{array}{ll}
Z_{u} & Z_{u} \\
Z_{u} & Z_{u}
\end{array}\right], Z_{A r}=\left[\begin{array}{ll}
Z_{u r} & Z_{u r}
\end{array}\right] . \\
& Y_{A}=\left[\begin{array}{cc}
Y_{c} & -Y_{c s} \\
-Y_{c s} & Y_{s}
\end{array}\right], Y_{A B}=\left[\begin{array}{cc}
0 & 0 \\
0 & -Y_{u}
\end{array}\right], Y_{A r}=\left[\begin{array}{ll}
0 & Y_{u r}
\end{array}\right] .
\end{aligned}
$$

where both the rows and columns of the matrices in Equations (8) and (9) are arranged in the order of the core, the shield. $Z_{\mathcal{C}}$ and $Z_{S}$ are the self-impedances of the core and the shield, respectively. $Z_{\mathcal{S} S}$ is the mutual impedance between the core and the shield. $Z_{u}$ is the mutual impedance between the cores or shields of two different subsystems. $Z_{u r}$ is the mutual impedance between the core (or the shield) and the armor. $Y_{c}$ and $Y_{s}$ are the self-admittances of the core and the shield, respectively. $Y_{c s}$ is the mutual admittance between the core and the shield. $Y_{u}$ is the mutual admittance between the shields of two different subsystems. $Y_{u r}$ is the mutual admittance between the shield and the armor.

The ATP-EMTP (Version 6.0, NTNU/SINTFE, Trondheim, Norway, 2015) software provides a routine named Cable Constants to calculate the p.u.l. parameters of cables [42]. This routine was 
adopted in this work. For example, the p.u.l. impedance and admittance matrices of the YJV22-type cable at $300 \mathrm{kHz}$ are shown in Appendix B.

The first-order phasor equations in Equation (1) can be expressed in the form of second-order ordinary differential equations:

$$
\left\{\begin{array}{c}
\frac{\mathrm{d}^{2}}{\mathrm{~d} x^{2}} V(x)=\operatorname{ZYV}(x), \\
\frac{\mathrm{d}^{2}}{\mathrm{~d} x^{2}} \mathbf{I}(x)=\operatorname{YZI}(x) .
\end{array}\right.
$$

To solve the differential equations, the actual phasor line voltages and currents are transformed to modal quantities, as described in Reference [13]:

$$
\left\{\begin{aligned}
V(x) & =Q V^{m}(x), \\
I(x) & =S \boldsymbol{I}^{m}(x) .
\end{aligned}\right.
$$

where $V^{m}$ and $I^{m}$ are the modal voltage vector and the modal current vector, respectively. The complex matrices $Q$ and $S$ are the corresponding modal transformation matrices, which define changes in the variables between the actual phasor line voltages and currents, and the modal voltages and currents.

Substituting Equation (11) into Equation (10) gives:

$$
\left\{\begin{array}{c}
\frac{\mathrm{d}^{2}}{\mathrm{~d} x^{2}} \boldsymbol{V}^{m}(x)=\boldsymbol{Q}^{-1} \boldsymbol{Z} \boldsymbol{Y Q} \boldsymbol{V}^{m}(x)=\boldsymbol{H}^{2} \boldsymbol{V}^{m}(x), \\
\frac{\mathrm{d}^{2}}{\mathrm{~d} x^{2}} \boldsymbol{I}^{m}(x)=\boldsymbol{S}^{-1} \boldsymbol{Y Z S \boldsymbol { I } ^ { m }}(x)=\boldsymbol{H}^{2} \boldsymbol{I}^{m}(x) .
\end{array}\right.
$$

where $Q$ and $S$ are the matrices chosen to diagonalize $Z Y$ and $Y Z$, respectively, via similarity transformations. $\boldsymbol{H}=\operatorname{diag}\left(\gamma_{1}, \gamma_{2}, \cdots, \gamma_{7}\right)$, where the diagonal elements are the eigenvalues of the matrices $Z Y$ and $Y Z$, or are known as the propagation constants of the modal quantities. Then, Equation (12) has a simple solution, transforming the solution back to the actual line voltages and currents via Equation (11) gives:

$$
\left\{\begin{array}{c}
\boldsymbol{V}(x)=\boldsymbol{Z}_{c} S\left(\boldsymbol{I}_{f}^{m} e^{-\boldsymbol{H} x}+\boldsymbol{I}_{b}^{m} e^{\boldsymbol{H} x}\right), \\
\boldsymbol{I}(x)=S\left(\boldsymbol{I}_{f}^{m} e^{-\boldsymbol{H} x}-\boldsymbol{I}_{b}^{m} e^{\boldsymbol{H} x}\right) .
\end{array}\right.
$$

where subscripts $f$ and $b$ represent the forward and backward modal quantities, respectively. $\mathbf{Z}_{c}=\mathbf{Z S H}^{-1} \boldsymbol{S}^{-1}$ is the CIM of the uniform MV underground cable.

The MV underground cable can be viewed as having $2 \times 7$ ports, with seven ports at the sending end $(x=0)$ and seven ports at the receiving end $(x=l)$, where $l$ is the length of the cable. According to Equation (13), when one end of the underground cable is connected to the CIM, the access impedance matrix of the other end is equal to the CIM. In addition, the phasor voltages and currents at the sending end and the receiving end have the relationship [13] as:

$$
\left[\begin{array}{c}
V(l) \\
I(l)
\end{array}\right]=T\left[\begin{array}{c}
V(0) \\
I(0)
\end{array}\right]
$$

where $\boldsymbol{T}$ is the transmission matrix, and can be expressed as:

$$
\boldsymbol{T}=\left[\begin{array}{cc}
\boldsymbol{Q} \cosh (\boldsymbol{H} l) S^{\mathrm{T}} & -\boldsymbol{Q} \sinh (\boldsymbol{H} l) \boldsymbol{S}^{\mathrm{T}} \boldsymbol{Z}_{c} \\
-\mathbf{Z}_{c}^{-1} \boldsymbol{Q} \sinh (\boldsymbol{H} l) S^{\mathrm{T}} & \mathbf{Z}_{c}^{-1} \boldsymbol{Q} \cosh (\boldsymbol{H} l) S^{\mathrm{T}} \boldsymbol{Z}_{c}
\end{array}\right]
$$

\section{Characteristic Impedance Matrices with Grounding Points}

For safe operation, the shields and armors of the MV underground cables are grounded at the RMUs, resulting in the whole cable structure being non-uniform, such that the above uniform transmission line model is no longer valid. In this section, the CIMs of the entire underground cable containing the grounding points are provided based on an eigen analysis of the overall transmission 
matrix of this non-uniform structure. The shields and armors are assumed to be grounded with grounding resistances only at the RMUs, of which the positions are arbitrary.

As described, the whole cable structure between the transceivers is divided into $k$ sections by the grounding points, an example being when $k=3$, as shown in Figure 2. Here, the three phase subsystems of the MV underground cables are drawn vertically to show the grounding structure more clearly.

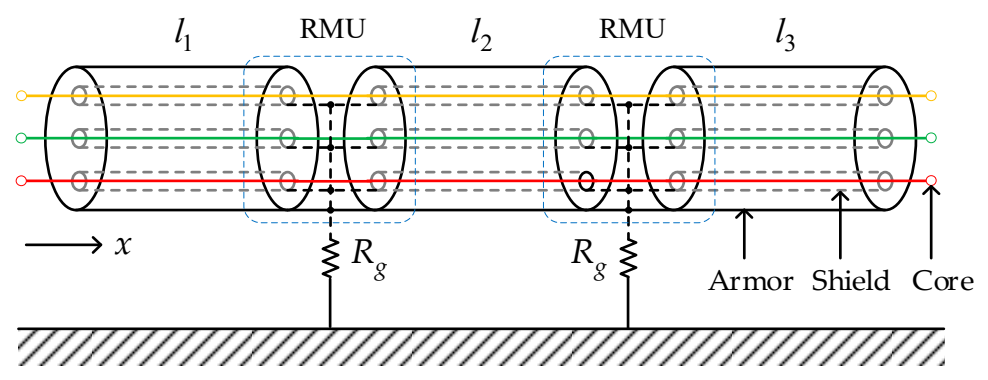

Figure 2. Structure of medium-voltage underground cables with shields and armors grounding at the ring main units (RMUs).

Assuming that the type and length of each section is arbitrary, and the total length is $L$, the voltage and current vectors at the sending end $(x=0)$ and the receiving end $(x=L)$ have the following relations:

$$
\left[\begin{array}{c}
\boldsymbol{V}(L) \\
\boldsymbol{I}(L)
\end{array}\right]=\overline{\boldsymbol{T}}\left[\begin{array}{c}
\boldsymbol{V}(0) \\
\boldsymbol{I}(0)
\end{array}\right]=\prod_{j=1}^{k-1}\left(\boldsymbol{T}_{j} \boldsymbol{T}_{g}\right) \boldsymbol{T}_{k}\left[\begin{array}{c}
\boldsymbol{V}(0) \\
\boldsymbol{I}(0)
\end{array}\right],
$$

where:

$$
\bar{T}=\left[\begin{array}{ll}
A & B \\
C & D
\end{array}\right]
$$

where $\bar{T}$ is the overall transmission matrix, and $A, B, C$ and $D$ are the sub-blocks. $T_{j}$ is the transmission matrix of the $j$ th cable section $(j=1,2, \cdots, k)$, which can be calculated from Equation (15). $\boldsymbol{T}_{g}$ is the transmission matrix of the grounding structure, which can be written as:

$$
T_{g}=\left[\begin{array}{cc}
E & 0 \\
-Y_{i n} & E
\end{array}\right]
$$

where $Y_{i n}=\operatorname{diag}\left(0,0,0, G_{g}, G_{g}, G_{g}, G_{g}\right)$ is the input admittance matrix of the grounding structure, $G_{g}=1 / R_{g} ; R_{g}$ is the grounding resistance; and $E$ is the $7 \times 7$ identity matrix.

If the positive direction of the current is the direction flowing into the port, then the impedance matrix $\overline{\mathbf{Z}}$ of the entire cable structure can be expressed as:

$$
\left[\begin{array}{c}
V(0) \\
V(L)
\end{array}\right]=\overline{\mathbf{Z}}\left[\begin{array}{c}
\boldsymbol{I}(0) \\
-\boldsymbol{I}(L)
\end{array}\right]=\left[\begin{array}{ll}
\bar{Z}_{11} & \bar{Z}_{12} \\
\bar{Z}_{21} & \bar{Z}_{22}
\end{array}\right]\left[\begin{array}{c}
\boldsymbol{I}(0) \\
-\boldsymbol{I}(L)
\end{array}\right],
$$

where:

$$
\left\{\begin{array}{c}
\bar{Z}_{11}=-C^{-1} D \\
\bar{Z}_{12}=-C^{-1} \\
\bar{Z}_{21}=B-A C^{-1} D \\
\bar{Z}_{22}=-A C^{-1}
\end{array}\right.
$$

The MV underground cable considered here is passive, thereby satisfying the condition of reciprocity, such that the impedance matrix is symmetrical: 


$$
\left\{\begin{array}{l}
\bar{Z}_{11}=\bar{Z}_{11}^{\mathrm{T}} \\
\bar{Z}_{12}=\bar{Z}_{21}^{\mathrm{T}}, \\
\bar{Z}_{22}=\bar{Z}_{22}^{\mathrm{T}} .
\end{array}\right.
$$

The eigen analysis of the overall transmission matrix $\bar{T}$ is based on the equation:

$$
\left[\begin{array}{cc}
A-\lambda E & B \\
C & D-\lambda E
\end{array}\right]\left[\begin{array}{l}
J \\
K
\end{array}\right]=\left[\begin{array}{l}
0 \\
0
\end{array}\right]
$$

where $\lambda$ is the eigenvalue of $\bar{T}$ and $\left[\begin{array}{ll}J & K\end{array}\right]^{\mathrm{T}}$ is the corresponding eigenvector. The elimination of $\boldsymbol{K}$ using the first equation in Equation (22) gives:

$$
\left[\lambda^{2} C^{-1}-\lambda\left(C^{-1} D+A C^{-1}\right)+A C^{-1} D-B\right] J=0 .
$$

Then, combining Equation (20) with Equation (23), it follows that:

$$
\left[\lambda^{2} \overline{\mathbf{Z}}_{12}+\lambda\left(\overline{\mathbf{Z}}_{11}+\overline{\mathbf{Z}}_{22}\right)+\overline{\mathbf{Z}}_{21}\right] \boldsymbol{J}=\mathbf{0}
$$

Equation (24) is then divided by $\lambda^{2}$ and transposed; so, it follows that:

$$
\left[\lambda^{-2} \overline{\mathbf{Z}}_{12}+\lambda^{-1}\left(\overline{\mathbf{Z}}_{11}+\overline{\mathbf{Z}}_{22}\right)+\overline{\mathbf{Z}}_{21}\right] \boldsymbol{J}=\mathbf{0} .
$$

Equations (24) and (25) indicate that both $\lambda$ and $\lambda^{-1}$ are the eigenvalues of $\bar{T}$, so the eigenvalue matrix $\Lambda$ of the overall transmission matrix $\bar{T}$ can be denoted as:

$$
\boldsymbol{\Lambda}=\operatorname{diag}\left(\boldsymbol{\Lambda}_{f}, \boldsymbol{\Lambda}_{b}\right)=\operatorname{diag}\left(\lambda_{1}, \lambda_{2}, \cdots, \lambda_{n}, \lambda_{1}^{-1}, \lambda_{2}^{-1}, \cdots, \lambda_{n}^{-1}\right) .
$$

where $\boldsymbol{\Lambda}_{f}$ and $\boldsymbol{\Lambda}_{b}$ are the $7 \times 7$ diagonal matrices, $\boldsymbol{\Lambda}_{b}=\boldsymbol{\Lambda}_{f}^{-1}$.

From the diagonalization of the overall transmission matrix $\bar{T}$, it follows that:

$$
\boldsymbol{P}^{-1} \overline{\boldsymbol{T}} \boldsymbol{P}=\left[\begin{array}{cc}
\boldsymbol{\Lambda}_{f} & \mathbf{0} \\
\mathbf{0} & \boldsymbol{\Lambda}_{b}
\end{array}\right], \boldsymbol{P}=\left[\begin{array}{ll}
Q_{f} & Q_{b} \\
S_{f} & S_{b}
\end{array}\right] .
$$

where $\boldsymbol{P}$ is the nonsingular matrix, and $\boldsymbol{Q}_{f}, \boldsymbol{Q}_{b}, S_{f}$ and $\boldsymbol{S}_{b}$ are the sub-blocks. Substituting Equation (27) into Equation (16) gives:

$$
\left[\begin{array}{c}
\boldsymbol{V}(L) \\
\boldsymbol{I}(L)
\end{array}\right]=\boldsymbol{P}\left[\begin{array}{cc}
\boldsymbol{\Lambda}_{f} & \mathbf{0} \\
\mathbf{0} & \boldsymbol{\Lambda}_{b}
\end{array}\right] \boldsymbol{P}^{-1}\left[\begin{array}{c}
\boldsymbol{V}(0) \\
\boldsymbol{I}(0)
\end{array}\right]
$$

or in the form of:

$$
\begin{gathered}
{\left[\begin{array}{c}
\boldsymbol{V}(x) \\
\boldsymbol{I}(x)
\end{array}\right]=\left[\begin{array}{ll}
\boldsymbol{Q}_{f} & \boldsymbol{Q}_{b} \\
\boldsymbol{S}_{f} & \boldsymbol{S}_{b}
\end{array}\right]\left[\begin{array}{l}
\boldsymbol{M}_{f}(x) \\
\boldsymbol{M}_{b}(x)
\end{array}\right],} \\
{\left[\begin{array}{c}
\boldsymbol{M}_{f}(L) \\
\boldsymbol{M}_{b}(L)
\end{array}\right]=\left[\begin{array}{cc}
\boldsymbol{\Lambda}_{f} & \mathbf{0} \\
\mathbf{0} & \boldsymbol{\Lambda}_{b}
\end{array}\right]\left[\begin{array}{l}
\boldsymbol{M}_{f}(0) \\
\boldsymbol{M}_{b}(0)
\end{array}\right] .}
\end{gathered}
$$

where $x=0$ or $x=L$. Compared to Equation (13), when $x=0$ or $x=L, M_{f}$ and $\boldsymbol{M}_{b}$ are the equivalent forward and backward modal quantities, respectively. $\Lambda_{f}$ and $\Lambda_{b}$ represent the relationships between them at both ends, so $\Lambda_{f}$ and $\Lambda_{b}$ can be denoted as:

$$
\left\{\begin{array}{c}
\Lambda_{f}=e^{-\overline{\boldsymbol{H}} x}, \\
\boldsymbol{\Lambda}_{b}=e^{\overline{\boldsymbol{H}} x} .
\end{array}\right.
$$


where $\overline{\boldsymbol{H}}=\operatorname{diag}\left(\bar{\gamma}_{1}, \bar{\gamma}_{2}, \cdots, \bar{\gamma}_{7}\right)$ is the equivalent propagation constant matrix. The voltage and current vectors at both ports can be represented by the equivalent modal currents, such that:

$$
\left\{\begin{array}{c}
\boldsymbol{M}_{f}(x)=\boldsymbol{I}_{f}^{m}(x), \\
\boldsymbol{M}_{b}(x)=-\boldsymbol{I}_{b}^{m}(x) .
\end{array}\right.
$$

where $\boldsymbol{I}_{f}^{m}$ and $\boldsymbol{I}_{b}^{m}$ are the equivalent forward and backward modal current vectors, respectively. By substituting Equation (32) into Equations (29) and (30), the voltage and current vectors are:

$$
\left\{\begin{array}{c}
\boldsymbol{V}(x)=\boldsymbol{Z}_{c, f} \boldsymbol{S}_{f} \boldsymbol{I}_{f}^{m} e^{-\overline{\boldsymbol{H}} x}+\boldsymbol{Z}_{c, b} \boldsymbol{S}_{b} \boldsymbol{I}_{b}^{m} e^{\overline{\boldsymbol{H}} x}, \\
\boldsymbol{I}(x)=\boldsymbol{S}_{f} \boldsymbol{I}_{f}^{m} e^{-\overline{\boldsymbol{H}} x}-\boldsymbol{S}_{b} \boldsymbol{I}_{b}^{m} e^{\overline{\boldsymbol{H}} x} .
\end{array}\right.
$$

where $x=0$ or $x=L$, and:

$$
\left\{\begin{array}{c}
Z_{c, f}=Q_{f} S_{f}^{-1} \\
Z_{c, b}=-Q_{b} S_{b}^{-1} .
\end{array}\right.
$$

Equation (33) has the same form as Equation (13). $S_{f}$ and $S_{b}$ are the transformation matrices of the forward and backward modal currents, respectively; $Q_{f}$ and $Q_{b}$ are the transformation matrices of the forward and backward modal voltages, respectively; and $\boldsymbol{Z}_{c, f}$ and $\boldsymbol{Z}_{c, b}$ are the forward characteristic impedance matrix (FCIM) and the backward characteristic impedance matrix (BCIM) of the whole cable structure, respectively. This indicates that the carrier signals at both ends of the MV underground cables with grounded shields and armor can still be regarded as the superposition of a series of modal quantities, as can the uniform MV underground cables without grounding points.

Equation (33) only applies to $x=0$ or $x=L$. There is no definite equivalence relation between $S_{f}$ and $\boldsymbol{S}_{b}$, or between $\boldsymbol{Z}_{c, f}$ and $\boldsymbol{Z}_{c, b}$, which indicates that the characteristic impedance matrices of the whole cable structure are different from those of one cable section. The former has two different characteristic impedance matrices, being the forward and backward impedance matrices, whereas the latter only has one unique characteristic impedance matrix. Additionally, the latter can be regarded as a special case of the former when the transformation matrices of the forward and backward modal quantities are equal.

Equation (33) shows that the access impedance matrix at the sending end is equal to $\boldsymbol{Z}_{c, f}$ only if the receiving end is connected to $\boldsymbol{Z}_{c, f}$. Similarly, the access impedance matrix of the receiving end is equal to $\boldsymbol{Z}_{c, b}$ only if the sending end is connected to $\boldsymbol{Z}_{c, b}$.

\section{Method Validation}

In this section, two examples are provided to validate the calculation of the characteristic impedance matrices of the whole cable structure between the PLC transceivers. Both examples possess a structure similar to that shown in Figure 2. Example 1 has three sections of cables, $l_{1}=100 \mathrm{~m}, l_{2}=300 \mathrm{~m}$ and $l_{3}=500 \mathrm{~m}$, whereas example 2 has two sections of cables, $l_{1}=l_{2}=300 \mathrm{~m}$. All the cables are of the YJV22-type MV underground cables described in Section 2, and the grounding resistances $\left(R_{g}\right)$ are $1 \Omega$.

As shown in Equation (33), if and only if the receiving end of the whole cable structure is connected to the FCIM, then the access impedance matrix of the sending end is equal to the FCIM. Similarly, if and only if the sending end of the whole cable structure is connected to the BCIM, then the access impedance matrix of the receiving end is equal to the BCIM. These characteristics were used to validate the calculation results of the FCIM and BCIM. For instance, take the validation of the FCIM in example 1. Firstly, the receiving end of the whole cable in example 1 was connected to the calculated FCIM. Then, the access impedance matrix of the sending end was measured. The calculated FCIM was validated if it was equal to the measured access impedance matrix. The principle for the validation of the BCIM was similar, the sending end of the whole cable was connected to the calculated BCIM, and the access impedance matrix of the receiving end was measured. 
The measurement setup is shown in Figure 3. Each cable section in examples 1 and 2 was simulated using 50 cascaded $\pi$ circuits, which were welded using lumped parameter components in the laboratory. The parameters of the $\pi$ circuits could be easily calculated using the p.u.l. parameters and the cable lengths. The receiving or sending end was connected to the lumped electrical networks where the impedance matrix was equal to the calculated FCIM or BCIM, respectively. These electrical networks are welded in the laboratory, and are labelled the FCIM network and BCIM network in this paper, respectively. Finally, the access impedance matrix of the sending or receiving end of the cables was measured using a HIOKI 3532-50 LCR meter. Given the multiport characteristics of the cables, the measurements were recorded for each port of the sending or receiving end. Each measurement was repeated three times, where the average value was taken as the measurement value.

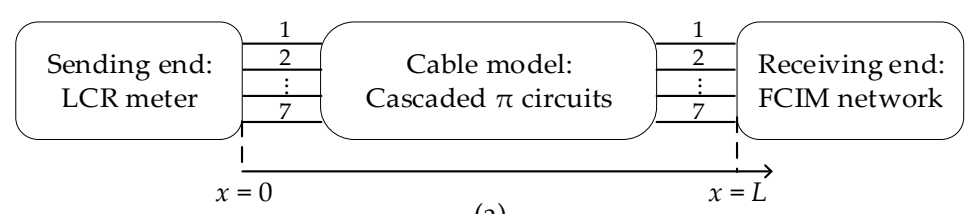

(a)

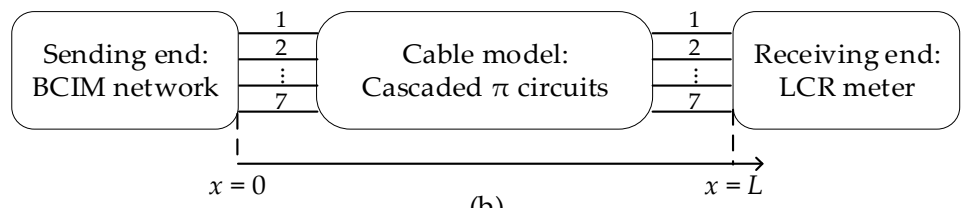

(b)

Figure 3. Measurement setup. The measurement of (a) the sending port's access impedance matrix and (b) the receiving port's access impedance matrix.

In this study, the FCIMs and BCIMs of two examples were calculated over the frequency of $10 \mathrm{kHz}-500 \mathrm{kHz}$, which was sampled into 246 frequency points with a sample interval of $2 \mathrm{kHz}$. For measurements, the above procedures were also carried out at some typical frequencies within the same frequency range. The selected frequencies included those at which the real or imaginary parts of the calculated characteristic impedances reached the local maximums or minimum values, the other frequency points were distributed evenly within the frequency ranges that did not contain the local maximum or minimum values.

In Figures 4 and 5, the solid lines represent the numerical results of the FCIM and BCIM for example 1. The crosses show the measurement results of the access impedance matrices of the sending and receiving ports. Specifically, $\boldsymbol{Z}_{c}(1,1), \boldsymbol{Z}_{c}(4,4)$ and $\boldsymbol{Z}_{c}(7,7)$ are the characteristic impedances of the core, the shield and the armor, respectively. $\mathbf{Z}_{c}(2,4)$ is the off-diagonal element in the characteristic impedance matrices, which results from the mutual impedance between the shields of the A-phase and B-phase.

Figure 4 shows that when the receiving port of the cable was connected to the calculated FCIM, the measured access impedance matrix of the sending port matched well with the calculated FCIM, indicating the credibility of the FCIM numerical results. Similar conclusions were obtained when comparing the numerical results and the measured results of the BCIM, as shown in Figure 5. At high frequencies, the differences between the numerical results and the measured results were larger than the differences at low frequencies. This happened because the approximation degree of the cascaded $\pi$ circuits decreased with increasing frequency.

For example 2, the numerical results are shown in Figure 6. The FCIM and BCIM in example 2 were the same. Then, the same measurements were recorded, and Figure 7 provides a comparation of the numerical results and the measured results, which also demonstrates the credibility of the numerical results of the characteristic impedance matrices. 

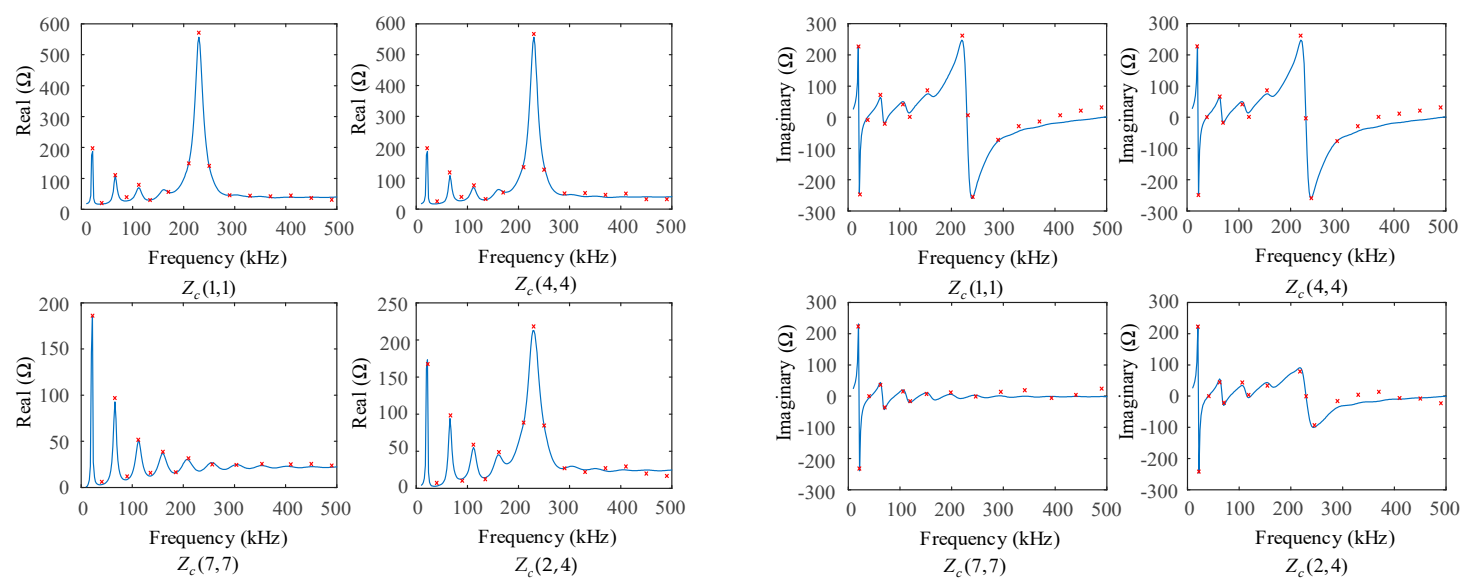

(a)
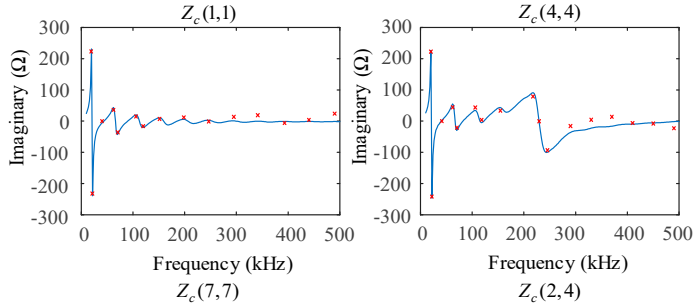

(b)

Figure 4. The validation of the forward characteristic impedances of medium-voltage underground cables with grounded shields and armor in example 1: (a) The real parts and (b) the imaginary parts of the characteristic impedances.
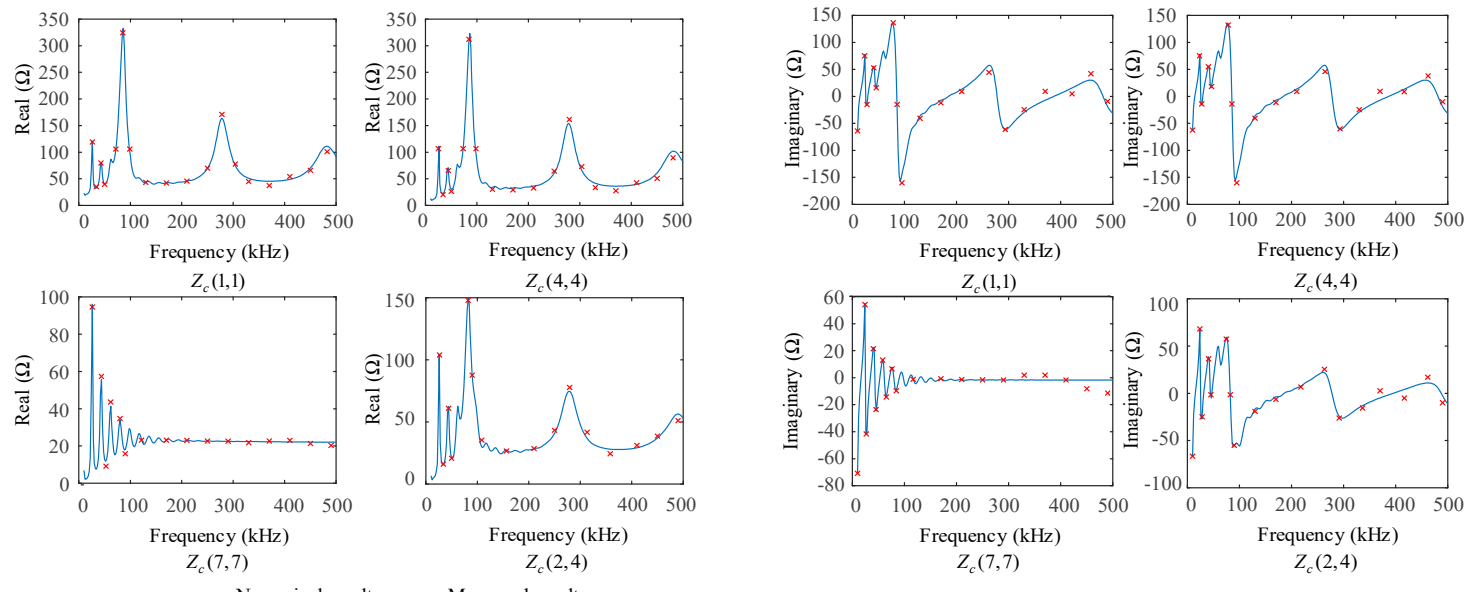

(a)

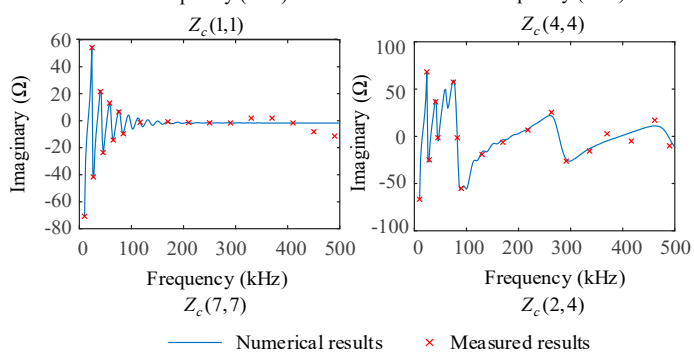

(b)

Figure 5. The validation of the backward characteristic impedances of medium-voltage underground cables with grounded shields and armor in example 1: (a) The real parts and (b) the imaginary parts of the characteristic impedances.
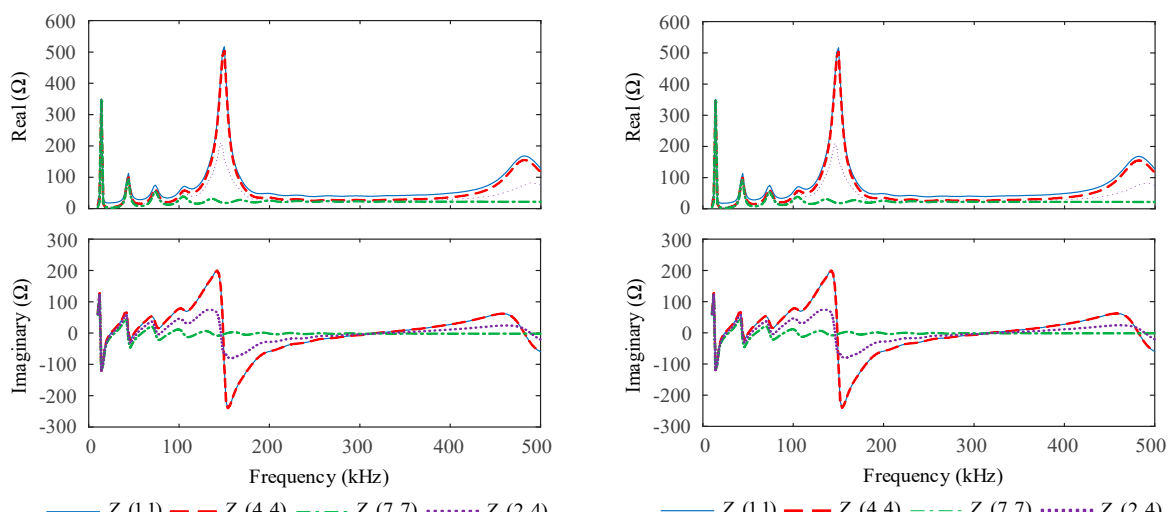

(a)

(b)

Figure 6. The characteristic impedances of medium-voltage underground cables with grounded shields and armor in example 2: (a) The numerical results and (b) the numerical results of the backward characteristic impedances. 

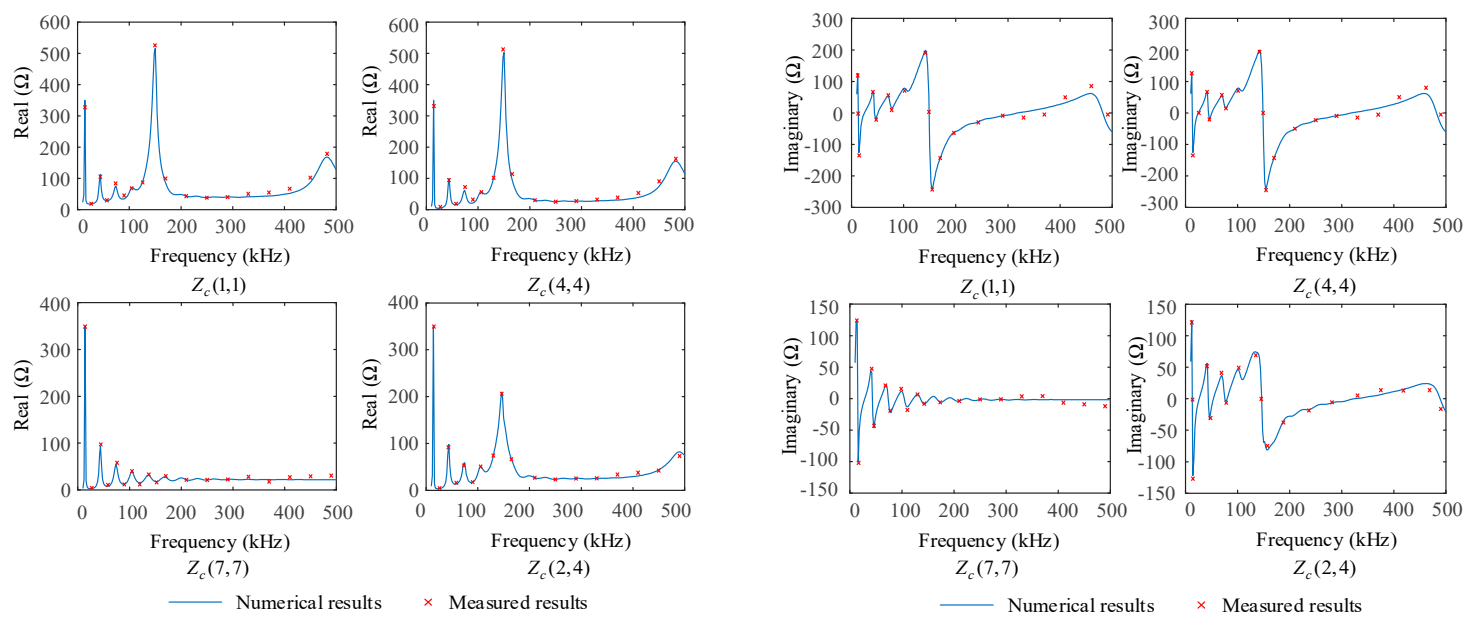

(a)

(b)

Figure 7. The validation of the forward characteristic impedances of medium-voltage underground cables with grounded shields and armor in example 2: (a) The real parts and (b) the imaginary parts of the characteristic impedances.

\section{Analysis of the Characteristic Impedance Matrices}

In this section, the characteristics of the FCIM and BCIM of the MV underground cables with grounded shields and armors are analyzed based on the results presented in Section 4, and on the investigation of Equations (33) and (34). To determine the effects of different factors (e.g., the high-frequency loss and grounding resistances), more experiments under different scenarios have been performed.

\subsection{Characteristics of the Forward and Backward Characteristic Impedance Matrices}

As shown in Figures 4-7, the MV underground cables with grounded shields and armor have FCIM and BCIM that are generally not equal, as shown in Equation (34). However, if the cable is longitudinally symmetrical, its FCIM and BCIM are equal, because the sending and receiving ends can be interchanged when the cable is longitudinally symmetrical.

Using the experimental results, we simultaneously checked that if the receiving end or sending end of the whole cable structure was connected to the FCIM or BCIM, the access impedance matrix at the other end was equal to the FCIM or BCIM. Combining these results with Equation (33), we concluded that if the receiving or sending port of the whole cable structure was connected with the FCIM or BCIM, the backward or forward modal quantities would be zero, and thus the signal power would be completely absorbed by the transceiver. Therefore, the characteristic impedance matrices of the whole cable structure between the transceivers should be considered to reduce or eliminate the reflected components of the carriers at the receivers or transmitters.

The characteristic impedances of the cores and shields were almost the same, indicating that the grounding of the shields not only affected their own characteristic impedances, but they also had a similar influence on the characteristic impedances of the cores covered by them owing to the mutual inductance between the cores and the shields.

Lastly, the results in Section 4 demonstrated that resonance occurred when the shields and armor of MV underground cable were grounded. At each resonant frequency, the real parts of the characteristic impedances showed high peaks, and the imaginary parts (phase angles) were zero, whereas the whole cable structure was pure resistive, as shown in Figures 4-7. These typical resonance phenomena signified that the electromagnetic waves at the resonant frequencies were standing waves, and the electromagnetic energy in the cables could not be transmitted, which hindered the transmission of carrier signals and adversely affected the impedance matching. 
To further study the above resonance characteristics, a control experiment was performed in which the characteristic impedance matrices from example 2 were calculated without the high-frequency loss. The results of the FCIM are shown in Figure 8, which shows that the characteristic impedances without the loss had a series of resonant points. The resonant frequencies were consistent with the frequencies in Figures 6 and 7, confirming the above judgment and indicating that the grounding of the shields and armor would lead to resonance, reflecting the multi-resonance characteristics. Figure 8 shows that the high-frequency loss had inhibitory effects on the above resonance, which made the curve around the resonance points smoother and reduces the resonant amplitudes. The higher the resonant frequency, the larger the high-frequency loss, and thus, the more evident the inhibitory effects.
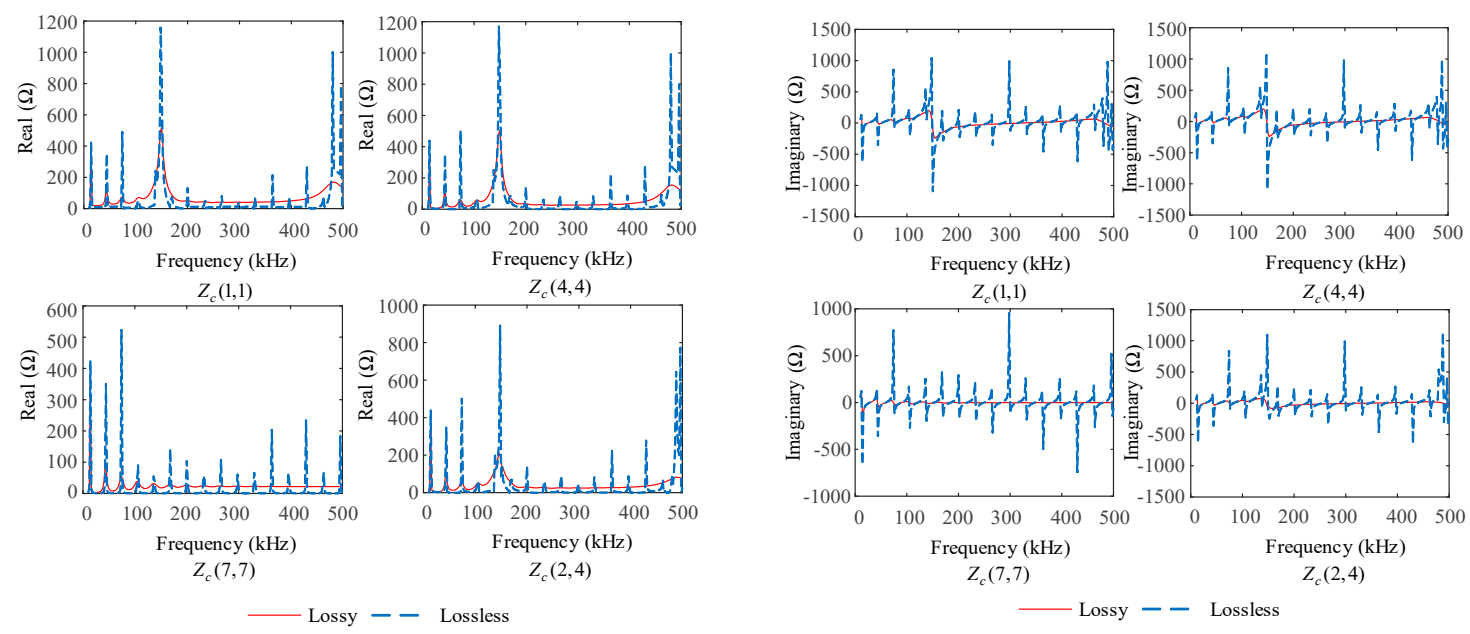

(a)

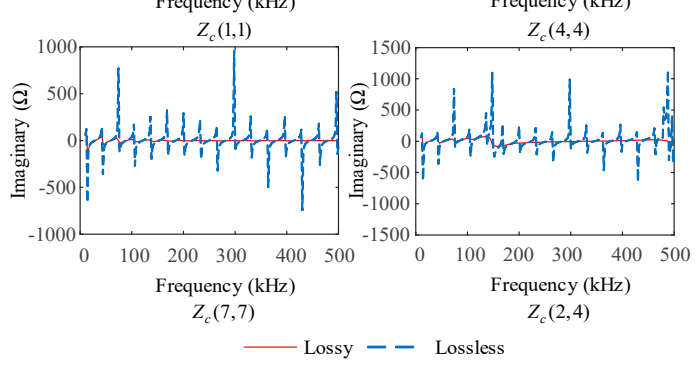

(b)

Figure 8. The comparation of the forward characteristic impedances when the cables are lossy and lossless: (a) The real parts and (b) the imaginary parts of the characteristic impedances.

\subsection{Effects of the Network Structures and Parameters}

It is fundamental for the PLC system to adapt to various power line networks. To demonstrate the effects of different MV network structures and parameters, a series of control experiments were conducted and are outlined in the following. The type and parameters of the cables used were the same as those for the MV underground cables used in Section 4.

\subsubsection{Effects of Grounding Resistances}

To analyze the effects of grounding resistances on the characteristic impedance matrices, the characteristic impedance matrices from example 1 with different grounding resistances $\left(R_{g}\right)$ were calculated.

The elements in the FCIMs are shown in Figure 9. When the shields and armor are ungrounded, then the cables can be regarded as uniform transmission lines, and thus there is no resonance in the characteristic impedances. When the shields and armor are grounded, the characteristic impedance matrices show resonance. Furthermore, the smaller the grounding resistance, the higher the peaks of the characteristic impedances at resonant frequencies. However, the differences in the grounding resistances do not affect the resonant frequencies.

\subsubsection{Effects of Cable Lengths}

To analyze the effects of the cable lengths on the characteristic impedance matrices, the characteristic impedance matrices from example 2 for different total cable lengths were calculated, assuming that the shields and armor were grounded at the half-length of the whole cables, $R_{g}=1 \Omega$.

The elements in the FCIMs are shown in Figure 10, which shows that the larger the total lengths of the cables, the more the resonant points shown by the characteristic impedances. Further calculations 
showed that all the electric lengths of the cables at the resonant frequencies were in the sequence of $0.087,0.147,0.211,0.296,0.945,1.607$, etc. This indicated that the resonant frequencies under the same structure were determined by the electric length of the whole cables, which was easy to understand considering the resonant characteristics. With increases in the cable lengths, the loss increases, the inhibition effect of high-frequency loss on the resonance gradually strengthens, and thus the peaks at the resonant frequencies gradually decrease.
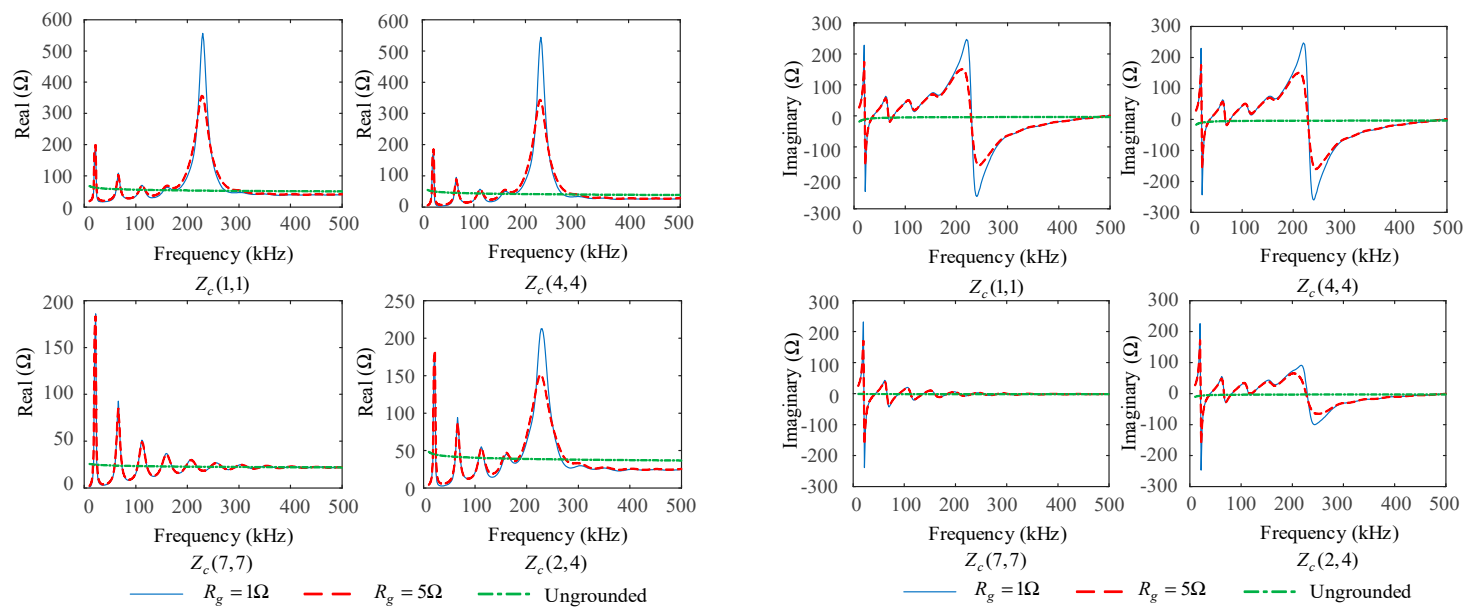

(a)

(b)

Figure 9. The forward characteristic impedances of medium-voltage underground cables with different grounding resistances: (a) The real parts and (b) the imaginary parts of the characteristic impedances.
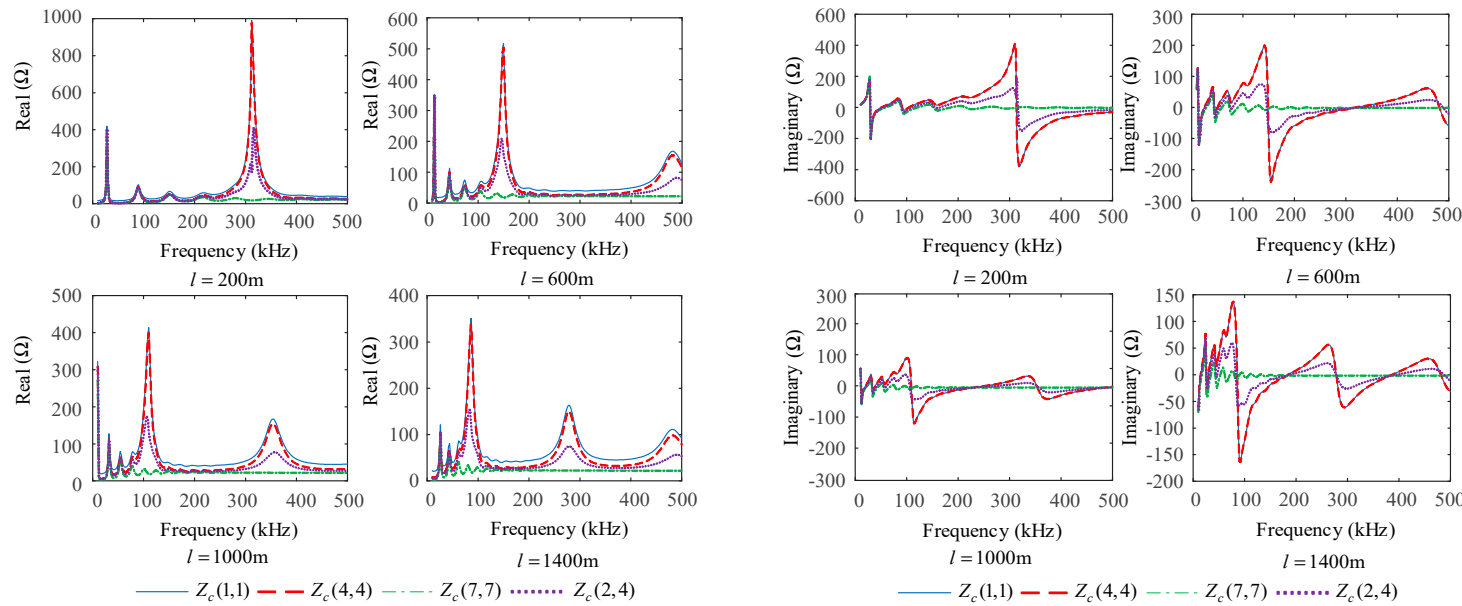

(a)

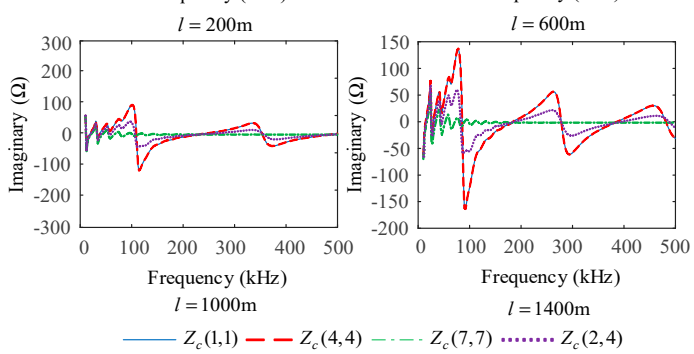

(b)

Figure 10. The forward characteristic impedances of medium-voltage underground cables with different cable lengths: (a) The real parts and (b) the imaginary parts of the characteristic impedances.

\subsubsection{Effects of the Grounding Point Numbers}

To analyze the effects of the grounding point numbers on the characteristic impedance matrices, the characteristic impedance matrices of the MV underground cables with $n_{g}$ grounding points were calculated, assuming that the grounding points were evenly distributed along the cables, where the total length of the cables was $600 \mathrm{~m}, R_{g}=1 \Omega$.

The elements in the FCIMs are shown in Figure 11, which shows that the larger the grounding point numbers, the fewer resonant points the characteristic impedances. Similar to the situation under different total cable lengths, at each resonant frequency, the electric lengths of the cables between the two grounding points were in the sequence of $0.046,0.077,0.112,0.160,0.171$, etc. With the decrease in 
cable length between two grounding points, the high-frequency loss decreases, so that the peaks at the resonant frequencies gradually increase.
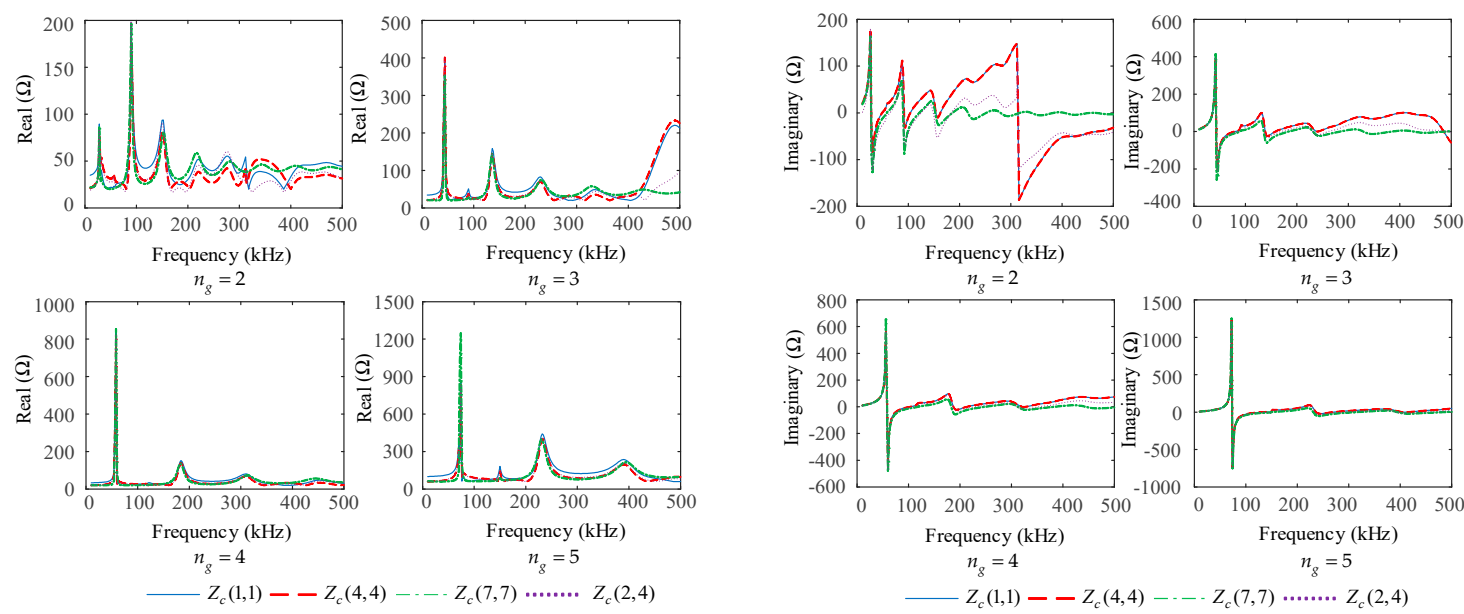

(a)

(b)

Figure 11. The forward characteristic impedances of medium-voltage underground cables with different grounding point numbers: (a) The real parts and (b) the imaginary parts of the characteristic impedances.

\subsubsection{Effects of Cable Branch Numbers}

To analyze the effects of the cable branches on the characteristic impedance matrices, the characteristic impedance matrices with $n_{b}$ cable branches were calculated assuming that the branches were also evenly distributed along the cables. Each cable branch was connected in parallel with one grounding branch, which is common in real-life scenarios. In this situation, the above calculation method was still valid, because the cable branch and grounding branch could easily be equivalent to one branch. The total length of the main cables was $600 \mathrm{~m}$, and $R_{g}=1 \Omega$. The branch cables were the same as the main cables, and the branches were well-matched to their characteristic impedance matrices.

The elements in the FCIMs are shown in Figure 12, where the results are similar to that in Figure 11, indicating that the effects of well-matched cable branches on the resonant frequencies are relatively small compared to those of the grounding points. This occurs because the relatively small impedance (i.e., the grounding impedance) plays a major role in the input impedance of the parallel structure. When the cable branches are connected in parallel to the grounding branches, the input impedances at the branch points decrease, and so the peaks in Figure 12 are higher than those in Figure 11, as revealed in Section 5.2.1.

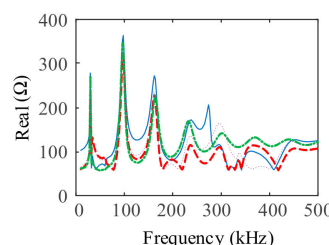

$n_{b}=2$

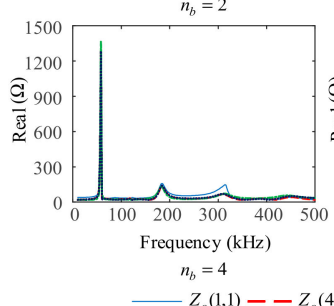

$n_{b}=4$

(a)

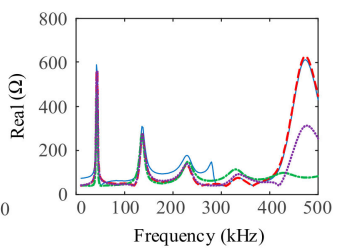

$n_{b}=3$

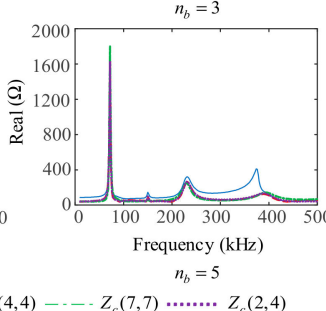

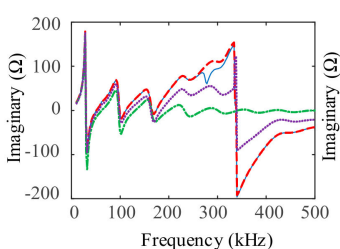

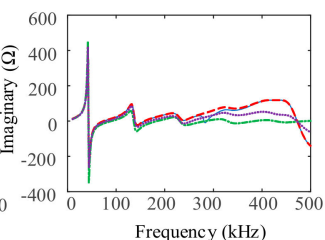

$n_{b}=2$
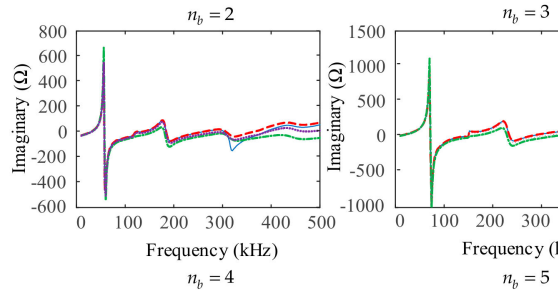

$n_{b}=3$

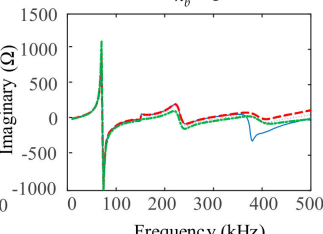

Frequency $(\mathrm{kHz})$

$-Z_{c}(1,1)--Z_{c}(4,4) \ldots . . Z_{c}(7,7) \cdots \cdots . . . Z_{c}(2,4)$

(b)

Figure 12. The forward characteristic impedances of medium-voltage underground cables with different branch numbers: (a) The real parts and (b) the imaginary parts of the characteristic impedances. 


\section{Conclusions}

At present, the calculations and analyses of MV underground cables as a power line communication channel are limited to studies under ideal conditions, where the MV underground cables are treated as uniform transmission lines. To consider more realistic situations, in this study, a non-uniform multiconductor transmission line model of MV underground cables was established with special consideration given to the grounded shields and armor. Then, the characteristic impedance matrices of the whole underground cables between the PLC transceivers were calculated through the eigen analysis of the overall transmission matrices. Thereafter, the characteristics of the FCIM and BCIM due to the grounding of the shields and armor were analyzed based on the numerical and measurement results. Lastly, the effects of the grounding resistances, line lengths, ground point numbers, and cable branch numbers on the characteristic impedance matrices were discussed. The main conclusions are as follows:

1. Considering the grounding of the shields and armors, the MV underground cables have FCIM and BCIM that were not equal unless the cables were longitudinally symmetrical. This is different from the case of uniform MV underground cables without grounding points, where the latter only has one unique characteristic impedance matrix.

2. If and only if the receiving end of the MV underground cable is connected to the FCIM, the access impedance matrix at the sending end is equal to the FCIM. Similarly, if and only if the sending end of the MV underground cable is connected to the BCIM, the access impedance matrix at the receiving end is equal to the BCIM.

3. The grounding of partial conductors will lead to resonance, resulting in multiple peaks in the characteristic impedances of the underground cables, which are not only reflected in the shields and armor, but can also be found in the cores. The resonance should be considered in investigations of the impedance matching for PLC to reduce or eliminate the reflected signal power at the transceivers.

4. The smaller the grounding resistance, the larger the amplitudes at the resonant points. The grounding resistance does not affect the resonant frequencies. The longer the cable, the more resonant points in the fixed frequency range, and the smaller the amplitudes at the resonant points. The more grounding points along the cable, the fewer resonant points in the fixed frequency range, and the larger the amplitudes at the resonance points. Compared to the grounding of the shields and armor, the effects of well-matched cable branches on the characteristic impedance matrices are limited.

The above conclusions are useful for understanding the impedance characteristics of MV underground cable and achieving impedance matching in PLC systems. In this study, the ground was modeled as a simple lumped resistance. However, the actual behavior of ground is more complex, and the grounding impedances may be different for different grounding points. The effects of these factors on the CIMs of MV underground cable will be investigated in future works.

Author Contributions: Conceptualization, Methodology and Validation, H.Z. and W.Z.; Investigation and Visualization, W.Z. and Y.W.; Writing-Original Draft Preparation, H.Z. and W.Z.; Writing-Review \& Editing, Supervision, H.Z. and Y.W.; Funding acquisition, Y.W.

Funding: This work was supported by the National Natural Science Foundation of China (Grant No. 51807063).

Acknowledgments: Thanks to the reviewers and editors for their careful review, constructive comments and English editing, which helped improve the quality of the paper.

Conflicts of Interest: The authors declare no conflict of interest.

\section{Appendix A}

The original geometric and electromagnetic parameters of the cable in this paper are provided in Table A1, where XPLE stands for cross-linked polyethylene, PVC stands for polyvinyl chloride. 
Table A1. Data sheet of the examined 10-kV underground cables (YJV22).

\begin{tabular}{cccc}
\hline Geometric Parameter & Radius $(\mathbf{m m})$ & Electromagnetic Parameter & Value \\
\hline Core conductor & 10.125 & Core or shield resistivity & $1.75 \times 10^{-8} \Omega \cdot \mathrm{m}$ \\
Cross-linked polyethylene & 15.325 & Armor resistivity & $9.78 \times 10^{-8} \Omega \cdot \mathrm{m}$ \\
(XPLE) insulation & 15.925 & Insulation relative permittivity & 2.6 \\
Copper shield & 42.050 & Sheath relative permittivity & 6 \\
$\quad$ Steel armor & 45.650 & Armor relative permeability ${ }^{1}$ & 1000 \\
Polyvinyl chloride & & & \\
(PVC) sheath & & & \\
\hline
\end{tabular}

${ }^{1}$ The relative permeability of mediums other than the armor is 1.

\section{Appendix B}

The per unit length parameter matrices at $300 \mathrm{kHz}$ of the medium-voltage underground cable examined in this paper are as follows:

$$
\begin{gathered}
\operatorname{Real}(\mathbf{Z})=\left[\begin{array}{cccccccc}
0.3812 & 0.3725 & 0.3539 & 0.3539 & 0.3539 & 0.3539 & 0.3311 \\
0.3725 & 0.3626 & 0.3539 & 0.3539 & 0.3539 & 0.3539 & 0.3311 \\
0.3539 & 0.3539 & 0.3812 & 0.3725 & 0.3539 & 0.3539 & 0.3311 \\
0.3539 & 0.3539 & 0.3725 & 0.3626 & 0.3539 & 0.3539 & 0.3311 \\
0.3539 & 0.3539 & 0.3539 & 0.3539 & 0.3812 & 0.3725 & 0.3311 \\
0.3539 & 0.3539 & 0.3539 & 0.3539 & 0.3725 & 0.3626 & 0.3311 \\
0.3311 & 0.3311 & 0.3311 & 0.3311 & 0.3311 & 0.3311 & 0.3311
\end{array}\right] \Omega / \mathrm{m}, \\
\operatorname{Imag}(\boldsymbol{Z})=\left[\begin{array}{cccccccc}
2.7228 & 2.5627 & 2.3966 & 2.3966 & 2.3966 & 2.3966 & 2.2424 \\
2.5627 & 2.5633 & 2.3966 & 2.3966 & 2.3966 & 2.3966 & 2.2424 \\
2.3966 & 2.3966 & 2.7228 & 2.5627 & 2.3966 & 2.3966 & 2.2424 \\
2.3966 & 2.3966 & 2.5627 & 2.5633 & 2.3966 & 2.3966 & 2.2424 \\
2.3966 & 2.3966 & 2.3966 & 2.3966 & 2.7228 & 2.5627 & 2.2424 \\
2.3966 & 2.3966 & 2.3966 & 2.3966 & 2.5627 & 2.5633 & 2.2424 \\
2.2424 & 2.2424 & 2.2424 & 2.2424 & 2.2424 & 2.2424 & 2.2424
\end{array}\right] \Omega / \mathrm{m} . \\
\boldsymbol{Y}=\left[\begin{array}{cccccccc}
0.90 i & -0.90 i & 0 & 0 & 0 & 0 & 0 \\
-0.90 i & 1.4 i & 0 & -0.20 i & 0 & -0.20 i & -0.20 i \\
0 & 0 & 0.90 i & -0.90 i & 0 & 0 & 0 \\
0 & -0.20 i & -0.90 i & 1.4 i & 0 & -0.20 i & -0.20 i \\
0 & 0 & 0 & 0 & 0.90 i & -0.90 i & 0 \\
0 & -0.20 i & 0 & -0.20 i & -0.90 i & 1.4 i & -0.20 i \\
0 & -0.20 i & 0 & -0.20 i & 0 & -0.20 i & 4.90 i
\end{array}\right] \mathrm{mS} / \mathrm{m} .
\end{gathered}
$$

\section{References}

1. Shaukat, N.; Ali, S.M.; Mehmood, C.A.; Khan, B.; Jawad, M.; Farid, U.; Ullah, Z.; Anwar, S.M.; Majid, M. A survey on consumers empowerment, communication technologies, and renewable generation penetration within Smart Grid. Renew. Sustain. Energy Rev. 2018, 81, 1453-1475. [CrossRef]

2. Chao, C.W.; Ho, Q.D.; Le-Ngoc, T. Challenges of power line communications for advanced distribution automation in smart grid. In Proceedings of the IEEE Power \& Energy Society General Meeting, Vancouver, BC, Canada, 21-25 July 2013; pp. 1-5.

3. Yang, L.; Crossley, P.A.; Wen, A.; Chatfield, R.; Wright, J. Design and performance testing of a multivendor IEC61850-9-2 process bus based protection scheme. IEEE Trans. Smart Grid 2014, 5, 1159-1164. [CrossRef]

4. Hadbah, A.; Ustun, T.S.; Kalam, A. Using IEDScout software for managing multivendor IEC61850 IEDs in substation automation systems. In Proceedings of the IEEE International Conference on Smart Grid Communications, Venice, Italy, 3-6 November 2014; pp. 67-72. 
5. Emmanuel, M.; Rayudu, R. Communication technologies for smart grid applications: A survey. J. Netw. Comput. Appl. 2016, 74, 133-148. [CrossRef]

6. Galli, S.; Scaglione, A.; Wang, Z. For the grid and through the grid: The role of power line communications in the smart grid. Proc. IEEE 2011, 99, 998-1027. [CrossRef]

7. Sauter, T.; Lobashov, M. End-to-end communication architecture for smart grids. IEEE Trans. Ind. Electron. 2011, 58, 1218-1228. [CrossRef]

8. Matanza, J.; Alexandres, S.; Rodríguez-Morcillo, C. Advanced metering infrastructure performance using European low-voltage power line communication networks. IET Commun. 2014, 8, 1041-1047. [CrossRef]

9. Milioudis, A.N.; Andreou, G.T.; Labridis, D.P. Detection and location of high impedance faults in multiconductor overhead distribution lines using power line communication devices. IEEE Trans. Smart Grid 2015, 6, 894-902. [CrossRef]

10. Della, G.D.; Ferrari, P.; Flammini, A.; Rinaldi, S.; Sisinni, E. Automation of distribution grids with IEC 61850: A first approach using broadband power line communication. IEEE Trans. Instrum. Meas. 2013, 62, 2372-2383.

11. De, P.M.; Tonello, A.M. On impedance matching in a power-line-communication system. IEEE Trans. Circuits Syst. II Express Briefs 2016, 63, 653-657.

12. Antoniali, M.; Tonello, A.M.; Versolatto, F. A study on the optimal receiver impedance for SNR maximization in broadband PLC. J. Electr. Comput. Eng. 2013, 2013, 1-11. [CrossRef]

13. Paul, C.R. Analysis of Multi-Conductor Transmission Lines, 2nd ed.; John Wiley and Sons: Hoboken, NJ, USA, 2007.

14. Costa, S.; de Queiroz, A.C.M.; Adebisi, B.; da Costa, V.L.R.; Ribeiro, M.V. Coupling for power line communications: A survey. J. Commun. Inform. Syst. 2017, 32, 8-22. [CrossRef]

15. Van Rensburg, P.A.; Sibanda, M.P.; Ferreira, H.C. Integrated impedance-matching coupler for smart building and other power-line communications applications. IEEE Trans. Power Deliv. 2015, 30, 949-956. [CrossRef]

16. Rui, C.P.; Barsoum, N.N.; Ming, A.W.; Ing, W.K. Adaptive impedance matching network with digital capacitor in narrowband power line communication. In Proceedings of the IEEE International Symposium on Industrial Electronics, Taipei, Taiwan, 28-31 May 2013; pp. 1-5.

17. Hallak, G.; Bumiller, G. Data rate optimization on PLC devices with current controller for low access impedance. In Proceedings of the International Symposium on Power Line Communications and its Applications (ISPLC), Bottrop, Germany, 20-23 March 2016; pp. 144-149.

18. Hallak, G.; Bumiller, G. Throughput optimization based on access impedance of PLC modems with limited power consumption. In Proceedings of the 2014 IEEE Global Communications Conference, Austin, TX, USA, 8-12 December 2014; pp. 2960-2965.

19. Rasool, B.; Rasool, A.; Khan, I. Impedance characterization of power line communication networks. Arab. J. Sci. Eng. 2014, 39, 6255-6267. [CrossRef]

20. Xiaoxian, Y.; Tao, Z.; Baohui, Z.; Fengchun, Y.; Jiandong, D.; Minghui, S. Research of impedance characteristics for medium-voltage power networks. IEEE Trans. Power Deliv. 2007, 22, 870-878. [CrossRef]

21. Cataliotti, A.; Daidone, A.; Tinè, G. Power line communication in medium voltage systems: Characterization of MV cables. IEEE Trans. Power Deliv. 2008, 23, 1896-1902. [CrossRef]

22. Yang, S.; Franklin, G. Effects of segmented shield wires on signal attenuation of power-line carrier channels on overhead transmission lines-Part I: Modeling method. IEEE Trans. Power Deliv. 2013, 28, 427-433. [CrossRef]

23. Yang, S.; Franklin, G. Effects of segmented shield wires on signal attenuation of power-line carrier channels on overhead transmission lines-Part II: Signal attenuation results analysis. IEEE Trans. Power Deliv. 2013, 28, 434-441. [CrossRef]

24. Araneo, R.; Celozzi, S.; Faria, J.A.B. Direct TD analysis of PLC channels in HV transmission lines with sectionalized shield wires. In Proceedings of the 2016 IEEE 16th International Conference on Environment and Electrical Engineering, Florence, Italy, 7-10 June 2016; pp. 1-4.

25. Franklin, G.A. Using modal analysis to estimate received signal levels for a power-line carrier channel on a 500-kV transmission line. IEEE Trans. Power Deliv. 2009, 24, 2446-2454. [CrossRef]

26. Wedepoh, L.M.; Mohamed, S.E.T. Multiconductor transmission lines: Theory of natural modes and fourier integral applied to transient analysis. Proc. Inst. Electr. Eng. 1969, 116, 1553-1563. [CrossRef] 
27. Wedepoh, L.M. Electrical characteristics of polyphase transmission systems, with special reference to boundary value calculations at power line carrier frequencies. Proc. Inst. Electr. Eng. 1965, 112, 2103-2112. [CrossRef]

28. Perz, M.C. Natural modes of power line carrier on horizontal three phase lines. IEEE Trans. Power Appl. Syst. 1964, 83, 679-686. [CrossRef]

29. Perz, M.C. A method of analysis of power line carrier problems on three-phase lines. IEEE Trans. Power Appl. Syst. 1964, 83, 686-691. [CrossRef]

30. Wedepoh, L.M.; Wasley, R.G. Wave propagation in polyphase transmission systems: Resonance effects due to discretely bonded earth wires. Proc. Inst. Electr. Eng. 1965, 112, 2113-2119. [CrossRef]

31. Faria, J.A.B.; da Silva, J.F.B. The effect of randomly earthed ground wires on PLC transmission: A simulation experiment. IEEE Trans. Power Deliv. 1990, 5, 1669-1677. [CrossRef]

32. Olsen, R.G. Propagation along overhead transmission lines with multiply grounded shield wires. IEEE Trans. Power Deliv. 2017, 32, 789-798. [CrossRef]

33. Rachidi, F.; Nucci, C.A.; Ianoz, M.; Mazzetti, C. Response of multiconductor power lines to nearby lightning return stroke electromagnetic fields. IEEE Trans. Power Deliv. 1997, 12, 1404-1411. [CrossRef]

34. Correia de Barros, M.T.; Festas, J.; Almeida, M.E. Lightning induced overvoltages on multiconductor overhead lines. In Proceedings of the International Conference on Power Systems Transients, Budapest, Hungary, 20-24 June 1999; pp. 365-369.

35. Andreotti, A.; Assante, D.; Verolino, L. Characteristic impedance of periodically grounded lossless multiconductor transmission lines and time-domain equivalent representation. IEEE Trans. Electromagn. Compat. 2014, 56, 221-230. [CrossRef]

36. Araneo, R.; Faria, J.M.; Celozzi, S. Frequency-domain analysis of sectionalized shield wires on PLC transmission over high-voltage lines. IEEE Trans. Electromagn. Compat. 2017, 59, 853-861. [CrossRef]

37. Faria, J.A.B.; Araneo, R. Computation, properties, and realizability of the characteristic immittance matrices of nonuniform multiconductor transmission lines. IEEE Trans. Power Deliv. 2018, 33, 1885-1894. [CrossRef]

38. Faria, J.A.B.; Araneo, R. Matching a nonuniform MTL with only passive elements is not always possible. IEEE Trans. Power Deliv. 2019, 34, 467-474. [CrossRef]

39. Benato, R.; Caldon, R. Distribution line carrier: Analysis and applications to DG. IEEE Trans. Power Deliv. 2007, 22, 575-583. [CrossRef]

40. Lazaropoulos, A.G.; Cottis, P.G. Broadband transmission via underground medium-voltage power lines-Part I: Transmission characteristics. IEEE Trans. Power Deliv. 2010, 25, 2414-2424. [CrossRef]

41. Ametani, A. A general formulation of impedance and admittance of cables. IEEE Trans. Power Appl. Syst. 1980, 99, 902-909. [CrossRef]

42. Dommel, H.W. Electromagnetic Transients Program (EMTP Theory Book); Bonneville Power Administration: Portland, OR, USA, 1986; pp. 150-191.

43. Schinzinger, R.; Ametani, A. Surge propagation characteristics of pipe enclosed underground cables. IEEE Trans. Power Appl. Syst. 1978, 5, 1680-1688. [CrossRef]

44. Amekawa, N.; Nagaoka, N.; Ametani, A. Impedance derivation and wave propagation characteristics of pipe-enclosed and tunnel-installed cables. IEEE Trans. Power Deliv. 2004, 19, 380-386. [CrossRef]

45. Wedepohl, L.M.; Wilcox, D.J. Transient analysis of underground power-transmission systems. System-model and wave-propagation characteristics. Proc. Inst. Electr. Eng. 1973, 120, 253-260. [CrossRef]

(C) 2019 by the authors. Licensee MDPI, Basel, Switzerland. This article is an open access article distributed under the terms and conditions of the Creative Commons Attribution (CC BY) license (http://creativecommons.org/licenses/by/4.0/). 